\title{
Rotating objects to determine orientation, not identity: Evidence from a backward-masking/dual-task procedure
}

\author{
STEFANO A. DE CARO and ADAM REEVES \\ Northeastern University, Boston, Massachusetts
}

\begin{abstract}
The effects of picture-plane rotations on times taken to name familiar objects (RTs) may reflect a process of mental rotation to stored viewpoint-specific representations: the rotate-to-recognize hypothesis. Alternatively, mental rotation might be used after stored object representations are activated by a misoriented stimulus in order to verify a weak or distorted shape percept: the double-checking hypothesis. We tested these two accounts of rotation effects in object recognition by having subjects verify the orientations (to within $90^{\circ}$ ) and basic-level names of 14-msec, backward-masked depictions of common objects. The stimulus-mask interval (SOA) varied from 14 to $41 \mathrm{msec}$, permitting interpolation of the SOA required for $75 \%$ accuracy $\left(\mathrm{SOA}_{c}\right)$. Whereas the $\mathrm{SOA}_{\mathrm{c}}$ to verify orientation increased with rotation up to $180^{\circ}$, the $\mathrm{SOA}_{c}$ to verify identity was briefer and asymptoted at $\sim 60^{\circ}$. We therefore reject the rotate-to-recognize hypothesis, which implies that $\mathrm{SOA}_{\mathrm{c}}$ should increase steadily with rotation in both tasks. Instead, we suggest that upright and near-upright stimuli are matched by a fast direct process and that misoriented stimuli are matched at a featural level by a slightly slower viewindependent process. We also suggest that rotation effects on RTs reflect a postrecognition stage of orientation verification: the rotate-to-orient hypothesis, a version of double-checking that also explains the well-known reduction in orientation effects on RTs when naming repeated objects.
\end{abstract}

The cognitive and perceptual mechanisms for visual object recognition in humans have evolved in an environment in which changes in viewing perspective are routine, reflecting our own mobility. Thus, the various perceptual constancies that have evolved (such as shape and size constancy) operate in an automatic, preconscious manner. But these same psychological mechanisms have also evolved in an environment in which objects tend to be upright. Indeed, shapes rotated in the two-dimensional (2-D) picture plane look rotated, suggesting there are no automatic, low-level mechanisms that compensate for the proximal effects of a "misoriented" figure. Shapes and objects misoriented in this manner therefore pose a potential problem for visual recognition that goes beyond the usual problem of perceptual constancy.

Successful and rapid recognition of misoriented objects imposes three processing requirements: bottom-up extraction of potentially useful stimulus information (stimulus components), access and matching to stored object representations, and selection and verification of the best

This work is based on a thesis presented by the first author in partial fulfillment of the requirements for the Master of Arts degree at Northeastern University. Portions of this paper were presented by the second author at the 36th Annual Meeting of the Psychonomic Society, Los Angeles. We thank Adam Friedman and Trang Le for running many of the subjects. We are also grateful to Thomas Sanocki and an anonymous reader for their careful reviews and encouraging comments. Correspondence concerning this article should be addressed to A. Reeves, Department of Psychology, Northeastern University, Boston, MA 02115 (e-mail: reeves@neu.edu). match. In this paper, we present relevant empirical data from object-naming experiments, summarize several current approaches to overcoming the effects of viewing geometry, and then describe how each approach can be applied (or even revised) to account for the data. Finally, we adopt a backward-masking procedure to isolate early stages of processing in order to test which approach is most likely to apply to the recognition of line drawings of common objects. We limit the problem of viewing geometry to recognizing unexpected (but common) objects depicted at unknown orientations in the picture plane, when location and approximate size are known. In support of the presupposition that extraction of orientation, location, and size are largely independent of each other, the magnitude of priming in object naming (as measured by the reduction in time to name a repeated object) is independent of whether there is a change in the object's location, size, or left-right orientation between the first and the second blocks (Biederman \& Cooper, 1991, 1992; E. E. Cooper, Biederman, \& Hummel, 1992). The restriction to common objects is made to ensure that recognition is based on stable, long-term basic-level categories in memory, not on evanescent representations derived from recently acquired nonsense figures.

\section{View Dependency in Object Naming}

There is now an extensive literature devoted to the recognition of common objects depicted at uncommon views. Empirical attempts to distinguish the various approaches have typically varied orientation in the 2-D picture plane, 
a transformation that does not change the visual information in the retinal image (unlike a rotation in depth). It is now well established that when adults name rotated depictions of common objects, verbal response times (RTs) tend to increase with increasing misorientation from the upright view (Jolicœur, 1985, 1988; Jolicœur \& Milliken, 1989; Maki, 1986; McMullen, Hamm, \& Jolicœur, 1995; McMullen \& Jolicœur, 1990; Murray, 1995, 1997). ${ }^{1}$ This well-known orientation effect is most pronounced with nonrepeated or novel objects, objects not previously presented to the subject (typically items presented in Block 1). If the objects are repeated even once (say, in Block 2), the orientation effect is dramatically reduced (Jolicœur, 1985) - so long as the objects were rotated in a previous block of trials (see Jolicceur \& Milliken, 1989). This diminution in viewpoint dependency is not a mere practice effect, because the initial orientation effect is restored when completely new objects are introduced later in the experiment (Jolicœur, 1985). We term the view dependency when naming novel objects and the reduction in view dependency when naming repeated objects the orientation reduction effect. ${ }^{2}$

\section{Overcoming Viewing Geometry in Recognition}

In the various image-centered approaches to recognition, projectively variant geometrical components are extracted from the proximal stimulus and then transformed (by rotation, scaling, or translation, as needed) to match an invariant representation in memory. Such geometrical components may be global (entire objects) or local (parts or features, such as corners and edges), depending on the approach. Computational models, such as the neocognitron neural network (Fukushima, Okada, \& Hiroshige, 1994), show that bottom-up transformations can compensate for unknown rotations and translations of an image that belongs to a known set of objects.

According to one popular image-centered approach, which we term the rotate-to-recognize hypothesis, the putative transformation is thought to involve (or at least mimic) mental rotation-a global rotation of the proximal stimulus (e.g., Jolicœur, 1985, 1988, 1990; Maki, 1986; Murray, 1997; Tarr \& Pinker, 1990, 1991). This hypothesis presupposes some form of guided rotation, however, in that the correct direction of rotation required to match each novel depiction must be known in advance; if it were not, the function relating mean RT to orientation would be flat. ${ }^{3}$ To explain the orientation reduction effect, one would also need to suppose that transforming a particular depiction speeds subsequent transformations of that depiction. However, the rate of mental rotation, as indexed by the time taken to locate the tops and bottoms of objects (McMullen \& Jolicœur, 1992) and by the time taken to discriminate left-facing and right-facing objects (Jolicour, 1985, 1988), is generally stable across blocks of trials. This latter point can be explained by Jolicœur's (1990) dual-route account, in which the orientation reduction effect reflects two subsystems, or routes, to object recognition: the first being rotate-to-recognize, the second being view-invariant. Orientation invariance might arise in any number of ways: The observer might learn to extract a subset of discerning features and parts that can be recovered from the image regardless of orientation (e.g., Biederman, 1987; Hoffman \& Richards, 1984); the features might be projectively invariant and so need no rotation (Rothwell, 1995); the features might be projectively variant but collectively require both clockwise and counterclockwise rotations, thus abrogating any global effect on RT; or a short-term memory might store copies of identifying features so that they need not be rotated again. In any case, the orientation reduction effect can be explained if the second route is slower than the first with novel object depictions but becomes faster with repeated depictions.

The multiple-views approach (e.g., Tarr \& Pinker, 1989) can partly explain the orientation reduction effect, since memory is assumed to include numerous prior views of each object, each view being of the proximal stimulus. Each time an object is viewed at an unfamiliar orientation, a new view-specific representation is stored in memory. Although memory storage is sacrificed, with sufficient prior views the visual system need not compensate for the projection at all. Rather, viewing geometry is implicitly overcome in memory. However, interpolation requires a fairly fine mesh of stored views in order to be effective, so it cannot explain the orientation effects for novel objects or for objects seen only in a few poses. Thus, Tarr and Pinker (1989) assume that the normalization of projectively variant stimulus components supplements interpolation.

Whereas the dual-route account attributes the orientation reduction effect to fewer normalizations, the multipleviews account attributes it to smaller normalizations; but both require some process, such as mental rotation, to explain how normalization is achieved. The doublechecking hypothesis of Corballis (1988) also attributes the orientation reduction effect to fewer normalizations. However, Corballis sketched a theory of shape recognition that relies on orientation-free descriptions (descriptions of shapes that are independent of any coordinate system). The theory assumes that the effects of stimulus orientation manifest at a relatively late stage of processing, when object-centered shape descriptions are established. Although these shape descriptions are sufficient for locating an object's internal axes (and sufficient for guiding normalization), misoriented shapes are likely to elicit only crude levels of activation in memory. Consequently, mental rotation is sometimes used to doublecheck the identity information derived from orientationfree descriptions. The idea that mental rotation is used to strengthen identification was also suggested by Maki (1986). Since there should be less need to verify a repeated object than a novel one, the double-checking hypothesis also explains the orientation reduction effect.

The projectively invariant components approach can explain the orientation reduction effect in a similar manner. In this approach, recognition is mediated by abstract geometrical properties (such as cross-ratios of image contour inflection points) extracted from the proximal stim- 
ulus, and their immediate compensation for viewing geometry permits direct access to memory by indexing (Rothwell, 1995). For unique matches, this approach correctly predicts that variations in orientation will have little effect on recognition until occlusions occur, when recognition will fail abruptly, as was illustrated in Biederman (1987). However, if more than one candidate object in memory is compatible with the extracted set of invariant components, a time-consuming back-projection process is needed to verify the match. This requires, for each of the candidates, reconstruction of a Euclidean representation from the invariant features stored in memory, followed by projection of this representation back onto the proximal stimulus - a process that is delayed when the current view is misoriented. If object matches are stored temporarily in a working memory, there is no back-projection and verification process for repeated items, and so the effect of view should diminish with repetitions of the same object.

In the object-centered approach, projectively variant components (such as surface or edge features) are extracted in early vision and then used to create an invariant volumetric visual reconstruction for matching to a canonical three-dimensional representation in memory (Marr, 1982; Marr \& Nishihara, 1978). To the extent that this is performed successfully, the memory-matching process relies on an invariant representation and will not be affected by viewing geometry. However, the finding that retinal projection-not the volumetric representation-is the deciding factor in old-new pattern recognition (Rock, 1983; Rock \& DiVita, 1987; Rock, DiVita, $\&$ Barbeito, 1981) and other evidence that viewing geometry has a marked effect on recognition (e.g., Hummel, 1994) cast some doubt on the volumetric theory.

\section{Evaluating the Various Accounts}

The orientation reduction effect is consistent to some degree with each of the theoretical accounts outlined above. However, it is not clear how the dual-route and double-checking (verification) accounts can explain why there is no diminution in view dependency when identifying objects repeatedly shown at a standard view and then shown at an unexpected view, as in Tarr and Pinker's (1989) experiments. The effects of orientation diminish only after the subject has identified rotated versions of the objects. If the novel depictions are all upright in Block 1, there is no diminution in view dependency when rotated versions are then introduced in Block 2 (Jolicœur \& Milliken, 1989). Presumably, the orientation reduction effect would not be observed in this situation until Block 3. Of the various theories described so far, only the multiple-views approach (Tarr \& Pinker, 1989) can explain this point without revision: Since upright depictions only reinforce canonical views in memory, there are no other views in memory until rotated objects have been introduced. ${ }^{4}$

The dual-route account can be amended by assuming that the view-dependent subsystem normally dominates recognition until rotated objects are introduced, after which point the second, view-invariant subsystem begins to support recognition. There is then no need to assume that the second subsystem is slower than the first with novel objects but faster with repeated objects to explain the orientation reduction effect. The second subsystem is presumed to be intrinsically faster but simply not available for novel objects.

The double-checking account may also have to be revised to be entirely consistent with the orientation reduction effect. Recently, Lawson and Jolicœur (1998) have challenged Corballis's (1988) double-checking hypothesis on the grounds that it lacks direct evidence and is unparsimonius, as compared with the dual-route account. They tested double-checking, using an unspeeded pictureword matching task and brief backward-mask depictions of common objects. On each trial, subjects were probed with three names, one of which correctly identified the masked object. Lawson and Jolicœur reasoned that presenting the correct match on every trial and removing pressure to respond rapidly should minimize doublechecking. Yet the results showed a clear effect of orientation on the number of correct matches; Lawson and Jolicour therefore rejected the double-checking hypothesis in favor of the dual-route account. However, in their case the effect of rotation in the plane asymptoted at $60^{\circ}$, which is not compatible with standard accounts of mental rotation. Progressive effects of orientation were observed when the distractor probes referred to objects that were visually similar to the depicted object. But forcing subjects to make more difficult identity matches should increase the need to verify those matches. Only when items are essentially random or unique are double-checking and rotation effects minimized, so it is not clear that these data oppose the double-checking hypothesis.

It may also be premature to reject double-checking in light of discrepancies in the effects of orientation on other measures of object recognition. For example, Hamm and McMullen (1998) used a word-picture matching task and varied the level of classification required to verify a match. The time required to verify objects against subordinate labels (e.g., collie) increased with rotation in the picture plane up to $\pm 120^{\circ}$, at a rate similar to that found in object naming, but basic-level (e.g., dog) and superordinate-level (e.g., animal) matches showed little or no effects of rotation on RTs. ${ }^{5}$ These results lend some support to the double-checking account, because humans routinely classify objects at the basic level when asked to name them (Snodgrass \& Vanderwart, 1980), and in most cases, the "basic level" corresponds to the level of categorization that is first accessed in memory (Jolicour, Gluck, \& Kosslyn, 1984). ${ }^{6}$ Presumably, the orientation effects in object naming arise because objects are encoded at a higher level of specificity (Murray, 1998), even though the objects are ultimately named at the basic level. ${ }^{7}$ Thus, double-checking may be a prominent signature of subordinate-level matching, when objects cannot be classified solely in terms of global shape. This could explain the results of Lawson and Jolicour (1998), but it cannot explain why, in one condition, the effects of orientation 
were limited to the first $60^{\circ}$ of rotation. One would expect the need for double-checking to be greater with objects rotated even farther from the upright (say, from $60^{\circ}$ to $180^{\circ}$ ). There is a solution to this point within the alternative framework of double-checking (see the Discussion section).

Corballis (1988) supposed that the purpose of doublechecking is to verify the object's identity, but we propose that some aspect of its geometry, such as global shape or orientation, might also be the focus of double-checking. Since the emergence of the orientation reduction effect requires the introduction of rotated objects, this implies that the increase in time to name objects depicted at unexpected views is directly related to a change in object orientation. In this paper, we argue that mental rotation (or some other time-consuming and geometrically similar process) is used to determine the orientation of a newly misoriented object, as part of a back-projection process of verification: the rotate-to-orient hypothesis.

\section{Rotate-to-Orient Hypothesis}

The rotate-to-orient hypothesis assumes that (1) the effects of plane rotations on object naming emerge after projectively invariant components are extracted from the proximal stimulus and matched to a canonical representation in memory, and (2) the effects of plane rotations reflect a process like mental rotation that is used to establish or verify the object's orientation. Such a two-step process may be required because a "misoriented" object introduces a conflict between perception and what is expected, given the orientation in which the object is typically seen. Indeed, Rock $(1973,1974,1983)$ illustrated the powerful effects of expected and perceived orientation on both phenomenal shape and recognition.

A distorted or weak shape percept (as introduced, for example, by a rotation in the plane) might signal an accidental view, but it could also signal the presence of a different object - that is, a simple case of mistaken identity. However, accidents in viewpoint are common enough in the real world that the human observer might first check orientation to verify the original interpretation of the object-rather than assume the interpretation was wrong. A time-consuming process such as mental rotation could be used to transform the proximal stimulus to match the view of a canonical representation in memory. The resultant transformation (the extent and direction of rotation needed to verify the match) would then signal the object's orientation relative to the observer.

\section{Mental Rotation and Judgments \\ About Orientation}

The rotate-to-orient hypothesis is tenable because mental rotation (Shepard \& Metzler, 1971) is demonstrated in tasks that explicitly require the subject to identify the orientations of rotated figures. For example, the time needed to judge whether a dot is located at the top or the bottom of an object increases as the object is rotated up to $180^{\circ}$ in the picture plane (McMullen \& Jolicœur, 1992). Similar results have been shown for front-behind decisions (Jolicœur, Ingleton, Bartram, \& Booth, 1993) or when subjects must decide whether figures rotated in the picture plane are left-facing or right-facing versions (Corballis \& Cullen, 1986; Jolicœur, 1988; McMullen \& Jolicour, 1990). Moreover, the effects of orientation in such tasks are robust over practice, even through six blocks of trials (see, e.g., Jolicœur, 1988). In L. A. Cooper and Shepard's (1973) classic experiments, similar effects of angular rotation on times required to discriminate normal and mirror-image versions of alphanumeric characters led to the hypothesis that subjects were mentally transforming misoriented characters back to the upright. In general, the observer might use a process like mental rotation to establish a common frame of reference with a rotated figure whenever the observer is trying to determine its orientation (Hinton \& Parsons, 1981).

The majority of orientation tasks used in psychology experiments have measured lateral (left-right) judgments, and so there are few cases in which subjects were explicitly required to determine orientation in the plane. ${ }^{8}$ Corballis, Zbrodoff, Shetzer, and Butler (1978) reported one experiment in which subjects viewed alphanumeric characters projected at various orientations on a screen. Half the subjects were required to verify the presence of a target character (e.g., the letter R), while ignoring orientation. The other subjects were required to verify the presence of a target orientation (e.g., any character rotated $60^{\circ}$ ), while ignoring character identity. The RTs required to verify orientation increased with rotation from the vertical axis and were longer than RTs to verify identity. De Caro (1998a) reported similar findings, using naturalistic objects and a word-picture verification procedure. Subjects viewed upright and rotated depictions of common objects and reported, as quickly as possible, whether the identity and orientation of the object matched a preceding description (such as upright car). The results showed that subjects were faster and more likely to correctly verify a mismatched object than a mismatched orientation, suggesting that subjects can recognize an object before classifying its orientation, at the lowest level of specificity, as either upright or rotated. More cognitive processing is required to determine orientation at higher levels of specificity (e.g., rotated 90 degrees), as was shown by Braine, Relyea, and Davidman (1981), so the time to determine how much an object is rotated in the plane should lag even further behind the time needed to identify the object.

Evidence for object recognition's occurring prior to knowledge of object orientation is consistent with the rotate-to-orient hypothesis, which assumes that orientation information is used to verify the recognition of rotated objects but is not required for accessing stored object representations in memory. In contrast, it is not clear how guided normalization, as required for rotate-to-recognize in both the dual-route and the multiple-views forms, can 
be implemented without prior knowledge of object orientation. The rotate-to-recognize hypothesis is further challenged by case studies of patients with impaired performance on mental rotation and orientation matching tasks but with intact recognition for rotated objects (Farah \& Hammond, 1988; Turnbull, Laws, \& McCarthy, 1995).

\section{Testing a Postrecognition Account of View Dependency with Backward Masking}

In general, both view-dependent and view-independentplus-checking hypotheses can predict that RTs to name misoriented objects will increase with rotation from a familiar view. Thus, some other operation is needed to distinguish these two accounts of view dependency in visual object recognition studies. To this end, we combined a picture verification task with backward masking, in which a brief presentation of the depicted object (the stim$u l u s$ ) is followed by a patterned stimulus of much longer duration (the mask). Varying the stimulus onset asynchrony (SOA), the time interval between stimulus and mask, can provide a method of studying perceptual mechanisms that operate at early stages of processing (Santee \& Egeth, 1982; Sperling, 1963), whereas RT reflects all the processes, including those that operate at late stages. Indeed, RTs in visual recognition tasks may be more sensitive to later processes, as can be illustrated by a direct comparison between RTs and SOAs for judgments of numerosity (Liss \& Reeves, 1983).

In this study, we are concerned only with the efficacy of backward masking in curtailing visual information processing at an early stage. Thus, we avoided random-dot masks, because they operate primarily at an early sensory (i.e., monoptic, possible retinal) level by stimulus degradation (Eriksen \& Lappin, 1964; Kinsbourne \& Warrington, 1962) and do not limit processing time (Turvey, 1973). We also avoided information masks, such as collages of superimposed objects, because these may interfere with recall (Bongartz \& Scheerer, 1976). Instead, we used patterned masks whose features (random line segments) were similar to those of the depicted objects, in terms of line thickness and distributions of orientations, spatial locations, and lengths. The masks were also high contrast and of long duration $(250 \mathrm{msec})$. These properties make it more likely that the mask will control stimulusprocessing time (Liss, 1968; Spencer \& Shuntich, 1970; Sperling, 1963) but will not affect subsequent recall.

By controlling visual information processing at a relatively early level, one can derive a critical SOA (denoted $\mathrm{SOA}_{\mathrm{c}}$ ), which is the SOA needed to reach a fixed level of performance. The dependency of $\mathrm{SOA}_{c}$ on orientation in the plane should help to identify the stage of processing (late vs. early) responsible for the effects of unfamiliar views on recognition performance.

We designed a dual-task procedure to obtain critical $\mathrm{SOA}_{c} \mathrm{~s}$ for verifying object identity and for verifying object orientation. The two tasks were equated to have a chance performance of $50 \%$ by using a two-alternative design. By combining backward masking with a dual- task procedure, we were able to test three predictions derived from the rotate-to-orient hypothesis. The first prediction is that the $\mathrm{SOA}_{\mathrm{c}}$ to verify object identity is shorter than the $\mathrm{SOA}_{\mathrm{c}}$ to verify object orientation, which follows if object recognition precedes the determination of orientation (Corballis, 1988; De Caro, 1998a, 1998b). This requires that the two tasks do not differ in their intrinsic difficulty, which we assessed in a no-mask control. The second prediction is that $\mathrm{SOA}_{c}$ will increase with rotation in the picture plane when the task is to verify orientation, which follows if a time-consuming process like mental rotation is used to determine orientation in the plane. The third prediction is that $\mathrm{SOA}_{\mathrm{c}}$ will be essentially independent of rotation when the task is to verify the basic-level identity of nonrepeated objects. This last prediction follows if mental rotation is not required for the recognition of misoriented novel objects, contra the rotate-to-recognize hypothesis. Instead, upright is a special case: If projectively invariant components are extracted and matched to a canonical representation, upright objects will be recognized earlier than rotated objects, but otherwise the degree of rotation from the upright should have little or no effect on $\mathrm{SOA}_{c}$.

\section{METHOD}

\section{Subjects}

The subjects in the main masking experiment were 48 undergraduate students who participated in partial fulfillment of their course requirements at Northeastern University. Twenty-four other subjects were tested in a control experiment with masking at a 0 msec SOA and with no mask. To be eligible for participation, the students were required to be native speakers of English and to demonstrate at least $20 / 30$ vision at the time of the experiment, as measured on a Snellen acuity scale. Visual acuity ranged from $20 / 15$ to $20 / 30$, with a median of $20 / 20$.

\section{Stimuli and Equipment}

The set of depicted objects consisted of 96 line drawings of common objects taken from the Snodgrass and Vanderwart (1980) picture set ( 89 items) and the Microsoft ClipArt gallery ( 7 items). Seven "misoriented" versions of each object were created by rotating the standard version in the picture plane in $45^{\circ}$ increments. This produced two sets of orthogonal views: a cardinal set $\left(0^{\circ}, 90^{\circ}, 180^{\circ}\right.$, and $\left.270^{\circ}\right)$ and an oblique set $\left(45^{\circ}, 135^{\circ}, 225^{\circ}\right.$, and $\left.315^{\circ}\right) .{ }^{9}$ The objects were depicted as black line drawings on a white background, using a 15 -in. ViewSonic monitor and $640 \times 480$ pixel resolution. The displays were controlled with an IBM-compatible 80486 (66 MHz) microcomputer and synchronized to an ATI mach32 graphics accelerator board operating at $72 \mathrm{~Hz}$ (resulting in a video frame rate of $13.8 \mathrm{msec}$ ). A chinrest was used to maintain a constant viewing distance of $56 \mathrm{~cm}$. At this distance, the circular mask subtended $10^{\circ}$ of visual angle, which was just large enough to cover the tallest and widest objects. There were eight versions of the mask, each consisting of lines and ares of random length and orientation.

\section{Groups}

The subjects were tested as two composite groups. This made it possible to collect adequate data, using nonrepeated objects. Half the subjects were tested with the set of four cardinal views; the other half were tested with the set of four oblique views. Thus, we limited the number of possible views to only four orthogonal orientations. It was necessary to equate the metric differences between any two 
orientations so that the level of orientation specificity was the same for all the subjects. Also, we wanted to maintain a reasonable level of specificity (namely, $90^{\circ}-180^{\circ}$ ), fearing that it might be difficult to accurately report smaller differences in orientation when objects are brief and masked. Both groups of subjects were tested with SOAs of 14,28 , and $41 \mathrm{msec}$.

\section{Basic-Level Verification Task}

Following the stimulus-mask sequence, the subjects were probed with the name of a familiar object and asked whether or not it correctly identified the preceding object. Mismatch names were randomly selected without replacement from a list of names of nondepicted objects. Match and mismatch object names are listed in the Appendix.

\section{Orientation Verification Task}

Following the stimulus-mask sequence, an arrow probe was displayed pointing in one of the four possible orientations. The subject's task was to respond yes if the top of the preceding object had been pointing in the same direction as the arrow and to respond no otherwise. (All 96 objects were judged beforehand to have unambiguous tops. Sixteen independent judges in a pilot study were asked to point to the top side of each object once with a computer mouse. An object was considered to have an unambiguous top if all the judges selected the same side of the object regardless of the object's orientation on the display.) Mismatch arrow probes were selected randomly, with the constraints that all four orientations were probed equally often across all trials and that the same mismatch probe was not used more than twice in any of the 3 (SOAs) $\times 4$ (views) $=12$ conditions.

\section{Procedure}

Each experimental trial began with the presentation of a black ring centered on an otherwise white display. The subject continued the trial by pressing a key, which blanked the display. The depicted object appeared $500 \mathrm{msec}$ later at the location previously occupied by the circle. Stimulus duration was held constant at $14 \mathrm{msec}$. After a predetermined SOA had elapsed, the masking stimulus appeared for $250 \mathrm{msec}$. The particular mask used on any given trial was determined randomly during the intertrial interval, with the constraint that the same mask could not be used on two successive trials. Two hundred and fifty milliseconds after mask offset, a probe stimuluseither the name of an object or an arrow - appeared on the screen. The onset of the second probe coincided with the subject's response to the first probe.

The type of probe (match or mismatch) used in each task on each trial was determined randomly, with the constraint that all four combinations of match and mismatch probes were used equally often in each of the 12 (SOA $\times$ view) conditions. Thus, regardless of SOA and view, correct responses were not correlated across the two tasks, and both tasks had the same guessing rate of $50 \%$. The order of the two tasks was fixed for each subject, but task order was counterbalanced across subjects within each orientation group. ${ }^{10}$ To counterbalance objects with conditions, a Latin-square design was used to ensure that each object was depicted in all 12 (SOA $\times$ view) conditions. No object was ever repeated to the same subject; thus, each subject participated in a single block of 96 trials.

\section{Practice}

The subjects completed a series of modified practice trials prior to the experiment. Sixteen alphanumeric characters were used as stimuli ( 12 letters and 4 digits). These stimuli were repeated in five blocks of decreasing SOA: no mask and 83, 41, 28, and $14 \mathrm{msec}$ (the 83-msec SOA introduced the mask without interfering with the perception of the stimulus). On each trial, the subject had to (1) identify the character by pressing its corresponding key on the computer keyboard and (2) indicate the orientation of the character by pressing one of four arrow keys. This practice session was used to familiarize the subject with backward masking and to clarify the type of identity and orientation information tested in the experiment.

\section{RESULTS}

\section{Preliminary Analyses}

Performance was measured as proportions of correct responses. All the statistical tests reported in this paper were based on arcsine-transformed scores, $y^{\prime}=2 \times$ $\sin ^{-1}(\sqrt{y}+1)$, which consistently resulted in greater homogeneity of variance than did untransformed scores. The $p$ values reported for effects involving withinsubjects factors were based on the Geisser-Greenhouse lower bound adjustment to degrees of freedom (e.g., Lewis, 1993). Figure 1 shows the psychometric functions relating overall task performance to SOA. Included in this graph are data from the control experiment, in which 24 subjects were tested with and without backward masking. Performance was at chance when the mask coincided with the stimulus ( $0-\mathrm{msec}$ SOA $)$ and near ceiling when the mask was omitted entirely. That performance was not perfect in the absence of backward masking can be attributed to the brief (14-msec) displays. In light of these results, we used .5 as the lower asymptote and forced the upper asymptote to 1 when fitting the psychometric functions to the data.

\section{General Analyses}

The individual scores from Experiment 1 were submitted to a mixed, three-way analysis of variance (ANOVA) to measure the effects of group (cardinal or oblique), task

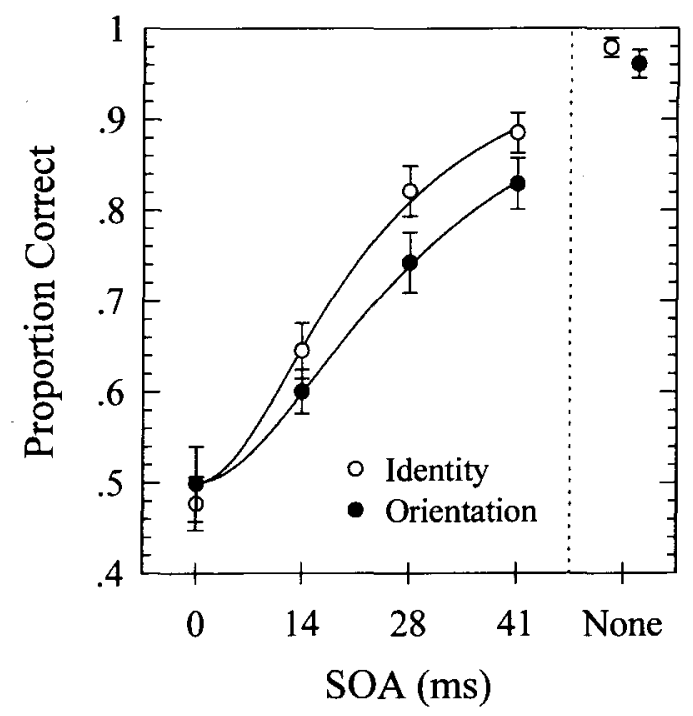

Figure 1. Mean proportion of correct responses in each task plotted as a function of stimulus onset asynchrony (SOA). Psychometric curves are best-fitting logistic functions, $y=0.5 /[1+$ $\left.(x / c)^{b}\right]+0.5$, where $c$ is the SOA corresponding to $75 \%$ correct and $b$ determines the slope. The data for the 0 -msec and no-mask conditions were obtained in a control experiment. Error bars are $\mathbf{9 5} \%$ confidence intervals. 
(identity or orientation), and SOA $(14,28$, or $41 \mathrm{msec})$ and their interactions. Only two main effects were significant. As is shown in Figure 1, report accuracy increased monotonically with SOA $[F(2,92)=169.03, p<.001]$ and varied with task $[F(1,46)=31.08, p<.001]$. The subjects were more likely to correctly verify the object's identity $(M=78.4 \%)$ than the object's orientation $(M=$ $72.4 \%$ ). There was a tendency for this difference to vary with SOA, but the effect was not reliable $[F(2,92)=2.25$, $p=.14]$. The performance of the cardinal and oblique orientation groups did not differ $(F<1)$, regardless of task and regardless of SOA $\left(F_{\mathrm{S}}<1\right)$. All these effects can be interpreted unambiguously, since there was no triple interaction between group, task, and SOA $(F<1)$.

Estimates of Critical Stimulus Onset Asynchronies. The $\mathrm{SOA}_{c} \mathrm{~s}$ were estimated by fitting the same logistic function used in Figure 1 to the mean data plotted in Figure 2. The values of the $\mathrm{SOA}_{c}$ were determined by the point of inflexion (denoted $c$ in the caption to Figure 1), which corresponded to $75 \%$ correct when the lower and upper asymptotes of the function were set to .5 and 1 , respectively. The $\mathrm{SOA}_{\mathrm{c}}$ to verify identity was estimated to be $21.6 \mathrm{msec}$; the $\mathrm{SOA}_{\mathrm{c}}$ to verify orientation was estimated to be $29.0 \mathrm{msec}$.

To assess the dependency of the $\mathrm{SOA}_{\mathrm{c}}$ on rotation in the plane, psychometric functions were also fit to each of the 2 (tasks) $\times 8$ (views) $=16$ data sets, which are plotted in Figure 2. The logistic function accounted for more than $95 \%$ of the variance in 15 cases (mean $R^{2}=.98$ ). The poorest fit resulted when the function was used to describe performance in the orientation task with objects depicted at $135^{\circ}$ views $\left(R^{2}=.92\right)$. The 16 estimates of $\mathrm{SOA}_{\mathrm{c}}$ derived from these data are plotted in Figure 3. It is clear that the effects of rotation on $\mathrm{SOA}_{\mathrm{c}}$ differed qualitatively in the two tasks. $\mathrm{SOA}_{c} s$ to verify orientation tended to increase with rotation from the upright, more
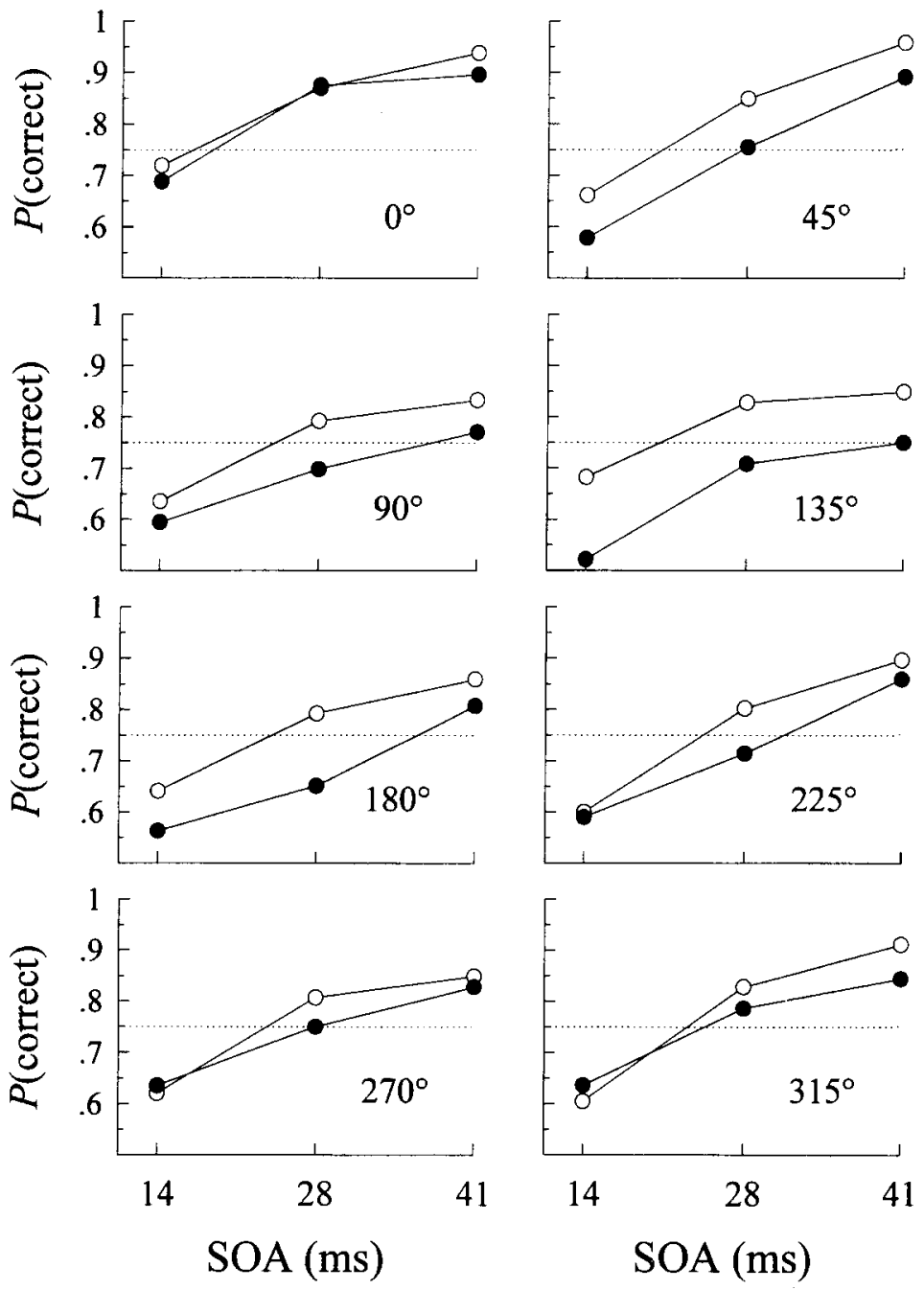

Figure 2. Mean proportion of correct responses in the identity task (open symbols) and orientation task (filled symbols) for each stimulus onset asynchrony (SOA) and view. 


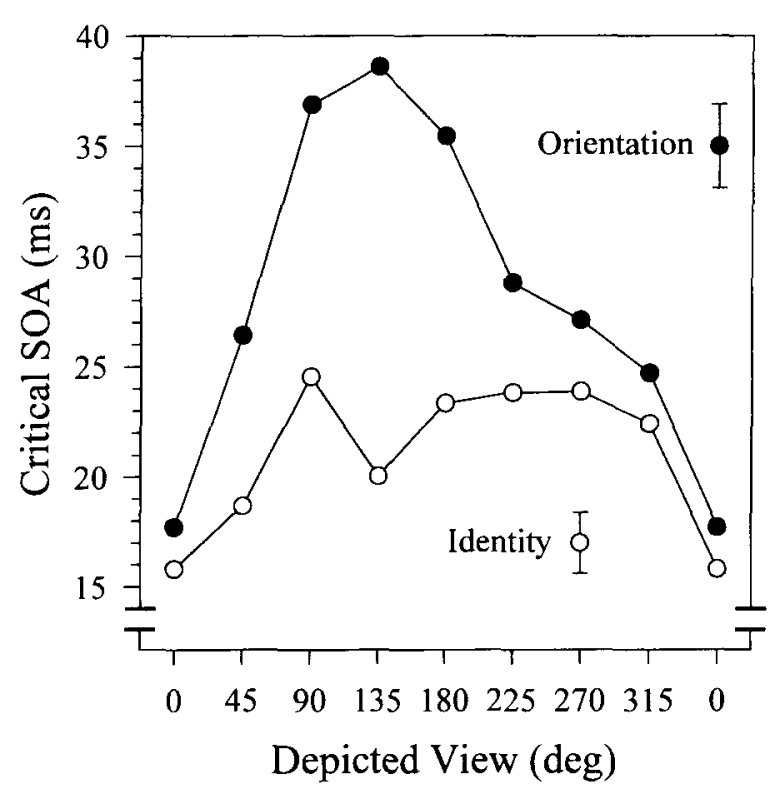

Figure 3. The estimated critical stimulus onset asynchronies (SOAs) for each task and view. Ordinate values correspond to $\mathbf{7 5 \%}$ correct performance. Error bars are the average standard error of each estimate, calculated separately for each task.

so when the object was rotated to the right of vertical $\left(45^{\circ}, 90^{\circ}\right.$, and $135^{\circ}$ views) than when it was rotated to the left of vertical $\left(225^{\circ}, 270^{\circ}\right.$, and $315^{\circ}$ views $) .^{11}$ In contrast, the effect of rotation on $\mathrm{SOA}_{\mathrm{c}}$ in the identity task was more dichotomous than linear. The $\mathrm{SOA}_{c}$ was shortest for upright $\left(0^{\circ}\right)$ views, but it varied only 6 msec across the $45^{\circ}-315^{\circ}$ range of misoriented views. Since these results agreed with the pattern of error rates listed in Table 1, we tested the linear trend, using contrast weights $-5,0,3,4,3,0$, and -5 applied to the error rates at $45^{\circ}$, $90^{\circ}, 135^{\circ}, 180^{\circ}, 225^{\circ}, 270^{\circ}$, and $315^{\circ}$, respectively. The results confirmed a progressive effect of rotation on performance in the orientation task $[F(1,184)=14.88, p<$ $.001]$ but no such effect on performance in the identity task $[F(1,184)=1.46, p=.23] .^{12}$

\section{DISCUSSION}

Both view-dependent and view-independent-pluschecking hypotheses predict that RTs to name misoriented objects will increase with rotation from a familiar view. In this study, we used backward masking by pattern as a method by which to distinguish these two accounts of view dependency in visual object recognition studies. The essential results are shown in Figure 3, in which the SOA required to reach a fixed level of performance, $\mathrm{SOA}_{\mathrm{c}}$, is plotted against spatial orientation. This figure shows three clear results: a table-top function to verify the basiclevel identity of nonrepeated objects, a $V$ function to verify object orientation, and an advantage for verifying identity over orientation. Plots for each spatial orientation (Figure 2) and summaries across orientations (Figure 1) confirm that this pattern is consistent and not an artifact of averaging.

From our own data in Figure 3 and from the results reported by Lawson and Jolicœur (1998), it appears that name verification is sensitive to the first $60^{\circ}$ of rotation from the upright, after which point the degree of rotation has little or no further effect on processing. Such a tabletop pattern for the verification of object identity rejects both pure view-dependent and pure view-independent processing. The outcome is, however, consistent with Rothwell's (1995) approach, outlined in the introduction, in which rotationally invariant features, such as cross ratios, are used to access candidate matches in memory. Provided there is more than one candidate match in memory, a time-consuming back-projection process is needed to verify which candidate is the best match, and this will depend on viewing geometry. An upright stimulus will match the best candidate directly, but a rotated stimulus will require matching particular geometric features, and this will take additional time. The time taken will depend on the details of the particular features and not on the global orientation of the stimulus relative to an external frame. Thus, a table-top pattern is expected.

The details of the table-top effect may prove important. In Figure 3, the difference between the $\mathrm{SOA}_{c}$ at $0^{\circ}$ $(16 \mathrm{msec})$ and the $\mathrm{SOA}_{\mathrm{c}}$ over the range of misoriented views (up to $25 \mathrm{msec}$ ) suggests that the time to match geometric properties of our objects is about $10 \mathrm{msec}$ longer than the time to match directly. (This estimate assumes that the random-line mask interferes with processing of upright depictions just as efficiently as it interferes with processing of rotated depictions and also that processing time is strictly limited by the mask; violations of these assumptions are unlikely to alter the order of magnitude of the estimate.) A mere 10-msec discrepancy between direct global matching and matching of geometric properties-although potentially important for theories of recognition--indicates that these different routes to early

Table 1

Mean Percentage of Errors in the Identity and Orientation Tasks for Each Type of View

\begin{tabular}{lcccccccc}
\hline & \multicolumn{8}{c}{ Depicted View of Object } \\
\cline { 5 - 9 } \multicolumn{1}{c}{ Task } & $0^{\circ}$ & $45^{\circ}$ & $90^{\circ}$ & $135^{\circ}$ & $180^{\circ}$ & $225^{\circ}$ & $270^{\circ}$ & $315^{\circ}$ \\
\hline Identity & 15.8 & 17.7 & 24.7 & 21.4 & 23.6 & 23.4 & 24.1 & 21.9 \\
Orientation & 18.1 & 25.9 & 31.3 & 34.0 & 32.6 & 28.0 & 26.2 & 24.5 \\
\hline
\end{tabular}

Note-Data are averaged across the 14-, 28-, and $41-\mathrm{msec}$ stimulus onset asynchrony conditions $(n=24$ per cell). 
recognition have almost the same latency in practice. The large increase in RTs for naming misoriented stimuli (of the order of 10ths of seconds) is likely to stem from postrecognition processes, such as verification of an unexpected orientation.

The progressive effect of orientation on $\mathrm{SOA}_{\mathrm{c}}$ (the $\mathrm{V}$ pattern) agrees with the rotate-to-orient hypothesis, in which orientation is checked by rotating the depiction along the shortest direction in the picture plane to the canonical upright. ${ }^{3}$ Since identity is already known and can be used to guide rotation, this hypothesis does not suffer from the logical paradox discussed by Corballis (1988) concerning the rotate-to-recognize hypothesis (i.e., having to assume that the direction of rotation is guided by knowledge of object identity before identity is established). The orientation verification data imply a rotation speed of about $10 \% \mathrm{msec}$, much faster than that assumed for the mental rotation of visual images but compatible with the fast processing speeds implied by some backwardmasking studies (Liss \& Reeves, 1983; Sperling, 1963). The data do not distinguish between a serial process in which a representation is literally transformed incrementally (from angle to angle) and parallel processes in which more time is required for rotated than for canonical images.

To the extent that verification judgments reflect underlying ascriptive processes, the pattern of results suggests that spatial orientation has quite different effects on the ascription of identity and the ascription of orientation. First, recall that both tasks were designed as yes-no verifications with the same chance level $(50 \%)$, to minimize task differences per se. The present findings therefore make it unlikely that global orientation is encoded before the object has been classified at the basic level. Second, objects were presented without repetition, a condition in which both the dual-route and the multiple-views accounts would predict large, progressive effects of misorientation. These points thus make it difficult to support the rotate-to-recognize hypothesis. Instead, the data show that identity is encoded first, and they suggest that if mental rotation is used, it is used to determine orientation (in our experiment) or to verify the recognition of a newly misoriented object (in Jolicœur, 1985) or of potentially confusable items (Murray, 1998). We therefore support view-independent-plus-checking (Corballis 1988)that is, postrecognition accounts of orientation encoding (De Caro, 1998a, 1998b). We also suggest that early recognition is supported by viewpoint-invariant processes, such as those proposed by Rothwell (1995).

Here, we should distinguish between explicit knowledge of global orientation (i.e., the orientation of an object's intrinsic axis relative to some external frame) and the implicit knowledge of global orientation resulting from knowledge of the orientation of features within the object and possibly of stable features in the environment (such as the edge of the display in our experiment). Such implicit knowledge may well be present early in visual processing. Indeed, it is plausible that the orientation of parts relative to other parts is known before all the parts of the depicted object are actually identified. Our claim is, rather, that the form of explicit knowledge required to guide normalization processes develops too late to support object identification at the basic level.

\section{REFERENCES}

BiedERMAN, I. (1987). Recognition by components: A theory of human image understanding. Psychological Review, 94, 115-147.

Biederman, I., \& CoOPER, E. E. (1991). Evidence for complete translational and reflectional invariance in visual object priming. Perception, 20, 585-593.

Biederman, I., \& Cooper, E. E. (1992). Size invariance in visual object priming. Journal of Experimental Psychology: Human Perception \& Performance, 18, 121-122.

Bongartz, W., \& SCheErer, E. (1976). Two visual stores and two processing operations in tachistoscopic partial report. Quarterly Journal of Experimental Psychology, 28, 203-291.

Braine, L. G., Relyea, L., \& Davidman, L. (1981). On how adults identify the orientation of a shape. Perception \& Psychophysics, 29, 138-144.

CoOper, E. E., Biederman, I., \& Hummel, J. E. (1992). Metric invariance in object recognition: A review and further evidence. Canadian Journal of Psychology, 46, 191-214.

COOPER, L. A., \& ShEPARD, R. N. (1973). The time required to prepare for a rotated stimulus. Memory \& Cognition, 1, 246-250.

Corballis, M. C. (1988). Recognition of disoriented shapes. Psychological Review, 95, 115-123.

Corballis, M. C., \& Cullen, S. (1986). Decisions about the axes of disoriented shapes. Memory \& Cognition, 14, 27-38.

Corballis, M. C., Zbrodoff, N. J., Shetzer, L. I., \& Butler, P. B. (1978). Decisions about identity and orientation of rotated letters and digits. Memory \& Cognition, 6, 98-107.

DE CARo, S. A. (1998a). On the perception of objects and their orientations. Spatial Vision, 11, 385-400.

DE CARo, S. A. (1998b). The timing of semantic and spatial representations in object recognition: Findings from an object-verification study. Unpublished doctoral dissertation, Northeastern University.

ERIKSEN, C. W., \& LAPPIN, J. S. (1964). Luminance summation-contrast reduction as a basis for certain forward and backward masking effects. Psychonomic Science, 1, 313-314.

FARAH, M. J., \& HAMMOND, K. M. (1988). Mental rotation and orientationinvariant object recognition: Dissociable processes. Cognition, 29, 2946.

Fukushima, K., OKada, M., \& Hiroshige, K. (1994). Neocognitron with dual C-cell layers. Neural Networks, 7, 41-47.

Hamm, J. P., \& MCMullen, P. A. (1998). Effects of orientation on the identification of rotated objects depend on the level of identity. Journal of Experimental Psychology: Human Perception \& Performance, 24, 413-426.

Hinton, G. E., \& Parsons, L. M. (1981). Frames of reference and mental imagery. In J. [B.] Long \& A. [D.] Baddeley (Eds.), Attention and performance IX (pp. 261-278). Hillsdale, NJ: Erlbaum.

Hoffman, D. D., \& Richards, W. A. (1984). Parts of recognition. Cognition, 18, 65-96.

Howard, I. (1982). Human visual orientation. New York: Wiley.

Hummel, J. E. (1994). Reference frames and relations in computational models of object recognition. Current Directions in Psychological Science, 3, 111-116.

Joliceur, P. (1985). The time to name disoriented natural objects. Memory \& Cognition, 13, 289-303.

JoLICEU, P. (1988). Mental rotation and the identification of disoriented objects. Canadian Journal of Psychology, 42, 461-478. 
JoLICGUR, P. (1990). Identification of disoriented objects: A dualsystems theory. Mind \& Language, 5, 387-410.

Joliceur, P., Gluck, M. A., \& Kosslyn, S. M. (1984). Pictures and names: Making the connection. Cognitive Psychology, 16, 243-275.

Jolicleur, P., Ingleton, M., Bartram, L., \& Booth, K. S. (1993). Top-bottom and front-behind decisions on rotated objects. Canadian Journal of Experimental Psychologv, 47, 657-677.

JOLICEUR, P., \& MILLIKEN, B. (1989). Identification of disoriented objects: Effects of context of prior presentation. Journal of Experimental Psychology: Learning, Memory, \& Cognition, 15, 200-210.

Kinsbourne, M., \& WarRington, E. K. (1962). The effect of an aftercoming random pattern on the perception of brief stimuli. Quarterly Journal of Experimental Psychology, 14, 223-234.

LAwson, R., \& Jolicaur, P. (1998). The effects of plane rotation on the recognition of brief masked pictures of familiar objects. Memory \& Cognition, 26, 791-803.

LEwIS, C. (1993). Analyzing means from repeated measures data. In G. Keren \& C. Lewis (Eds.), A handbook for data analysis in the behavioral sciences: Statistical issues (pp. 73-94). Hillsdale, NJ: Erlbaum.

Liss, P. (1968). Does backward masking by visual noise stop stimulus processing? Perception \& Psychophysics, 4, 328-330.

Liss, P., \& REEves, A. ( 1983). Interruption of dot processing by a backward mask. Perception, 12, 513-529.

MAKI, R. H. (1986). Naming and locating the tops of rotated pictures. Canadian Journal of Psychology, 40, 368-387.

MARR, D. (1982). Vision. San Francisco: Freeman.

MarR, D., \& Nishinara, H. K. (1978). Representation and recognition of the spatial organization of three-dimensional shapes. Proceedings of the Roval Society of London: Series B, 200, 269-294.

MCMULLEN, P. A. (1995). Identification of real world, rotated objects and orientation-specific templates. Paper presented at the Object Perception and Memory Symposium, Los Angeles.

McMullen, P. A., Hamm, J., \& Jolicaur, P. (1995). Rotated object identification with and without orientation cues. Canadian Journal of Psychology, 49, 133-149.

MCMullen, P. A., \& Joliceur, P. (1990). The spatial frame of reference in object naming and discrimination of left-right reflections. Memory \& Cognition, 18, 99-115.

McMullen, P. A., \& Jolicaur, P. (1992). Reference frame and effects of orientation on finding the tops of rotated objects. Journal of Experimental Psychology: Human Perception \& Performance, 18, 807-820.

MuRRAY, J. E. (1995). The role of attention in the shift from orientationdependent to orientation-invariant identification of disoriented objects. Memory \& Cognition, 23, 49-58.

MurraY, J. E. (1997). Flipping and spinning: Spatial transformation procedures in the identification of rotated natural objects. Memory \& Cognition, 25, 96-105.

MurRaY, J. E. (1998). Is entry-level recognition viewpoint invariant or viewpoint dependent? Psychonomic Bulletin \& Review, 5, 300-304.

Murray, J. E., Jolicceur, P., McMullen, P. A., \& Ingleton, M. (1993). Orientation-invariant transfer of training in the identification of rotated natural objects. Memory \& Cognition, 21, 604-610.

Rock, I. (1973). Orientation and form. New York: Academic Press.

Rock, I. (1974). The recognition of disoriented figures. Scientific American, 230, 78-85.

Rock, 1. (1983). The logic of perception. Cambridge, MA: MIT Press.

Rock, I., \& DiVITA, J. ( 1987). A case of view-centered object perception. Cognitive Psychology, 19, 280-293.

Rock, I., DiVita, J., \& Barbeito, R. (1981). The effect on form perception of change of orientation in the third dimension. Journal of Experimental Psychology: Human Perception \& Performance, 7, 719-732.

ROTHWELL, C. A. (1995). Object recognition through invariant indexing. Oxford: Oxford University Press.

SANTEE, J. L., \& EgETH, H. E. (1982). Independence versus interference in the perceptual processing of letters. Perception \& Psychophysics, 31, 101-116.

ShePARD, R. N., \& Metzler, J. (1971). Mental rotation of threedimensional objects. Science, 171, 701-703.
Snodgrass, J. G., \& VANDERWART, M. (1980). A standardized set of 260 pictures: Norms for name agreement, image agreement, familiarity, and visual complexity. Journal of Experimental Psychology: Human Learning \& Memory, 6, 174-215.

SPEnCER, T. J., \& ShUNTiCh, R. (1970). Evidence for an interruption theory of backward masking. Journal of Experimental Psychology, 85, 198-203.

SPERLing, G. (1963). A model for visual memory tasks. Human Factors, 5, 19-31.

TARr, M. J., \& Pinker, S. (1989). Mental rotation and orientationdependence in shape recognition. Cognitive Psychology, 5, 233-282.

TARR, M. J., \& Pinker, S. (1990). When does human object recognition use a viewer-centered reference frame? Psychological Science, 1, 253-256.

TARr, M. J., \& Pinker, S. (1991). Orientation-dependent mechanisms in shape recognition: Further issues. Psychological Science, 2, 207-209.

Turnbull, O. H., Laws, K. R., \& McCarthy, R. A. (1995). Object recognition without knowledge of object orientation. Cortex, $\mathbf{3 1}$, 387-395.

Turvey, M. T. (1973). On peripheral and central processes in vision: Inferences from an information-processing analysis of masking with patterned stimuli. Psychological Review, 80, 1-52.

Ullman, S. (1989). Aligning pictorial descriptions: An approach to object recognition. Cognition, 32, 193-254.

\section{NOTES}

1. Under certain conditions, $180^{\circ}$ rotations give rise to slightly faster performance than do $\pm 120^{\circ}$ rotations, resulting in an RT function that is somewhat M-shaped, rather than $\wedge$-shaped, across $360^{\circ}$ (see Murray, 1997).

2 . The terms practice effect and familiarity effect $t$ have been used in the literature. We use this more neutral designation to describe the effect without implying an explanation for it.

3. It has been argued that there are visual features that can convey information about an object's orientation without presupposing object identification - such as taper, salient protrusions, oriented surface texture, and axes of symmetry, elongation, and balance (e.g., Howard, 1982; Ullman, 1989) - but we question whether orientation is determined before identity (De Caro, 1998a). We also note that unguided rotation predicts that the function relating RT variance to orientation would have a minimum at $180^{\circ}$, but this function is essentially flat, if not more variable, at $180^{\circ}$. Thus, unguided mental rotation can be rejected on both counts, mean and variance.

4. Still, we are aware of some evidence suggesting that multiple views are brought to bear on recognition only when objects are visually similar (McMullen, 1995), and so the multiple-views account may not apply to the domain of naturalistic objects often represented in object-naming experiments (see also Murray, Jolicœur, McMullen, \& Ingleton, 1993).

5. The RTs to verify objects depicted at $180^{\circ}$ did not agree with this interpretation, but Hamm and McMullen (1998) argued that these data should be given less weight when measuring the slope of the orientation effect, because of the known variability in naming times at $180^{\circ}$.

6 . To account for the fact that atypical members of basic-level categories are sometimes identified at the subordinate level (e.g., as penguin rather than as bird), Jolicœur et al. (1984) use the term entry level to specifically refer to the first level accessed in memory; but for most items, the basic level is the entry level.

7. This raises a different kind of question-namely, why naming objects at the basic level would lead to additional processing at the subordinate level. A tenative explanation is that because object naming is an ostensive form of identification, there is a higher threshold for emitting a response than in the case of word-picture matching, which-being an indirect form of identification - might normally not require a verification process (as when targets and distractors are essentially random).

8 . In some cases, the subjects may have determined orientation in the plane even though the task required a simple polarity judgment. For example, a left-right decision might require the subject to first ascertain orientation in the plane, so that left and right can be defined with 
respect to the figure's known top-bottom axis, and not with respect to the subject's own body axes.

9. The angles of rotation refer to clockwise increments from the upright (see, e.g., Joliccur, 1985). Note that because the rotated depictions are static displays, any particular pose can be viewed as resulting from a rotation in either direction. For example, an object depicted at $270^{\circ}$ looks the same as one rotated $90^{\circ}$ counterclockwise.

10. We found no reliable effects of task order in this study.

11. We have noticed similar asymmetries routinely in the objectnaming literature, so that the function relating naming time to orientation is slightly steeper from $0^{\circ}$ to $180^{\circ}$ than from $180^{\circ}$ to $360^{\circ}$.
12. Since each subject was tested with only a subset of the seven misoriented views (e.g., $90^{\circ}, 180^{\circ}$, and $270^{\circ}$ ), the depicted view of the object was treated as a between-subjects factor.

13. Although we suggest that orientation might be checked in this manner, the observer might instead rotate an external (vertical) frame to match the object's internal axis. Both strategies imply a time cost that is proportional to the amount of stimulus misorientation, making it difficult to discern the two processes with our chronometric data. We have little reason to support one process over the other because, in either case, orientation information is presumably realized by the amount and direction of rotation required for a match.

APPENDIX

List of Match and Mismatch Object Names

\begin{tabular}{|c|c|c|c|c|c|}
\hline \multicolumn{3}{|c|}{ Match Names (Depicted Objects) } & \multicolumn{3}{|c|}{ Mismatch Names } \\
\hline airplane & football helmet & pineapple & anchor & goat & salt shaker \\
\hline alligator & fox & pitcher & ashtray & hairbrush & scarecrow \\
\hline ant & frog & purse & barrel & hair dryer & sea horse \\
\hline baby carriage & frying pan & pot & birdbath & harp & sewing machine \\
\hline barn & gift & rabbit & bottle & igloo & shark \\
\hline basket & garbage can & record player & bridge & joystick & sheep \\
\hline bear & giraffe & rhinoceros & bus & knight & skateboard \\
\hline bed & globe & rocking chair & bush & ladder & skirt \\
\hline bells & gorilla & sailboat & butterfly & lady bug & skunk \\
\hline bicycle & grasshopper & seal & cactus & lantern & sled \\
\hline bird & gun & shirt & camera & lawn mower & slide projector \\
\hline boot & hat & shoe & canoe & leopard & spinning wheel \\
\hline bowl & helicopter & snail & car & light house & suitcase \\
\hline cake & horse & snow man & castle & lobster & swing \\
\hline camel & house & squirrel & caterpillar & lock & table \\
\hline candle & iron & stapler & clock & mailbox & telephone booth \\
\hline cannon & ironing board & stool & coffee maker & microscope & telescope \\
\hline cat & kangaroo & stove & coat hanger & microwave oven & television \\
\hline chicken & kettle & swan & coat rack & paintbrush & tennis racket \\
\hline church & $\operatorname{lamp}$ & telephone & computer & park bench & tent \\
\hline clown face & light bulb & toaster & deer & parking meter & tiger \\
\hline coat & lion & traffic light & desk & peacock & toilet \\
\hline couch & monkey & tree & doll & pelican & train \\
\hline cow & motorcycle & truck & door & pipe & tricycle \\
\hline crown & mountain & turtle & dress & pumpkin & typewriter \\
\hline cup & mouse & vase & drum & pyramid & umbrella \\
\hline $\operatorname{dog}$ & mushroom & vest & duck & raccoon & vacuum cleaner \\
\hline donkey & ostrich & wagon & electric fan & refrigerator & water faucet \\
\hline dresser & owl & watering can & fire hydrant & roller skate & whale \\
\hline eagle & penguin & well & flag & rooster & whistle \\
\hline elephant & piano & wine glass & flower & row boat & windmill \\
\hline fish & pig & yacht & fly & saddle & zebra \\
\hline
\end{tabular}

(Manuscript received March 9, 1999;

revision accepted for publication December 7,1999 .) 